\title{
The Photovoltaic Mechanism of a Polythiophene/Perylene Pigment Two-Layer Solar Cell
}

\author{
Jun-ichi Nakamura, ${ }^{1,2}$ Shinji Suzuki, ${ }^{1}$ Kohshin Takahashi, ${ }^{* 1}{ }^{1}$ Chiho Yokoe, ${ }^{2}$ and Kazuhiko Murata ${ }^{2}$ \\ ${ }^{1}$ Division of Innovative Technology and Science, Graduate School of Natural Science and Technology, \\ Kanazawa University, Kakumamachi, Kanazawa 920-1192 \\ ${ }^{2}$ E \& I Materials Research Laboratory, Nippon Shokubai Co., Ltd., 5-8, Nishi Otabi-cho, Suita, Osaka 564-8512
}

Received March 17, 2004; E-mail: ktakaha@t.kanazawa-u.ac.jp

\begin{abstract}
The energy conversion efficiency of a two-layer organic solar cell consisting of a perylene pigment (PV) and regioregular polythiophene polymer (P3DT) was $0.99 \%$ under illumination with simulated solar light (AM 1.5-100 $\mathrm{mW} \mathrm{cm}{ }^{-2}$ ). The photovoltaic mechanism is discussed on the basis of the ionization potential of the component materials. The photoinduced hole-transfer from PV to P3DT quickly occurred at the PV/P3DT interface because of the large free energy change of $1.2 \mathrm{eV}$, then the subsequent charge separation efficiently proceeded, resulting in a large short-circuit photocurrent of $6.5 \mathrm{~mA} \mathrm{~cm}^{-2}$. On the other hand, the open-circuit photovoltage was only $0.42 \mathrm{~V}$ because the LUMO level of PV is close to the HOMO level of P3DT. The difference between the LUMO and the HOMO can be attributed to the open-circuit photovoltage.
\end{abstract}

Since Tang reported an energy conversion efficiency of up to $1 \%$ by using a phthalocyanine/perylene pigment $\mathrm{p}-\mathrm{n}$ heterojunction cell, ${ }^{1}$ much effort has been made to provide organic solar cells with low-cost and practical energy-conversion. However, the efficiency is still small because of the low light utility originating from the cell structure and the small carrier mobility of the organic materials. Further detailed investigations on the photovoltaic effect are necessary to fabricate practical organic solar cells. Recently, photovoltaics using conjugated polymers with relatively large mobility have been extensively studied. For example, Granström et al. attained an energy conversion efficiency of up to $1.9 \%$ using a cyano derivative of poly(phenylenevinylene)/polythiophene doublelayer solar cell under AM 1.5 illumination. ${ }^{2}$ Shaheen et al. attained an energy conversion efficiency of up to $3.3 \%$ in a $\mathrm{p}-\mathrm{n}$ hetero-bulk-junction solar cell consisting of soluble poly(phenylenevinylene) and fullerene 60 derivatives. ${ }^{3,4}$ In this case, light was used more effectively because the charge separation site increased by blending the component materials. In addition, the charge separation occurred smoothly due to the large built-in potential created by modifying two current-collecting electrodes with large work functional poly(ethylene dioxythiophene) doped with polystyrenesulphonic acid (PEDOT:PSS) and with low work functional $\mathrm{LiF}$, respectively. ${ }^{5}$ The built-in potential gradient of the $\mathrm{p}-\mathrm{n}$ hetero-bulk-junction is compensated because the micro-phase separated domains of the donor and the acceptor due to such blending are scattered in the single layer. On the other hand, Breeze et al. investigated on a ptype poly(phenylenevinylene) derivative/n-type perylene pigment two-layer solar cell and made the photovoltaic mechanism considerably clear. ${ }^{6}$ Other approachs for enhancing the photocurrent have been tried by co-depositing PV and copper phthalocyanine to increase the charge separation area, and further by annealing the co-deposited layer to make the carrier transport easy. ${ }^{7-10}$

In this work, we attempt to explain the photovoltaic mechanism in a polythiophene/perylene pigment two-layer solar cell. The elucidation of such a mechanism will contribute to finding promising materials for enhancing the power conversion yield of organic solar cells.

\section{Experimental}

Regioregular 3-alkyl polythiophenes P3DT and P3HT were purchased from Aldrich Chemicals, and perylene pigment PV from SYNTEC. The compounds were used without further purification. Porphyrins were synthesized and purified by a literature method. ${ }^{11}$ The chemical structures and the abbreviations of the compounds employed in this work are shown in Fig. 1.

ITO/In/PV/conjugated polymer/Au sandwich-type solar cells, shown in Fig. 1, were fabricated as follows. First, an In-layer of 5 $\mathrm{nm}$ thickness was prepared on a pre-cleaned indium-tin oxide (ITO) electrode by vacuum-deposition at $3 \times 10^{-5}$ Torr. The ITO electrode (sheet resistance: $8 \Omega / \square$ ) was purchased from Merck Display Technologies Ltd. Secondly, PV was vacuum-deposited at $3 \times 10^{-5}$ Torr on the ITO covered with the thin In-layer. Third, a chloroform polymer solution was spin-coated on the insoluble PV solid film. Finally, an Au electrode of $25 \mathrm{~nm}$ film thickness was prepared on the polymer film by vacuum-deposition at $8 \times 10^{-5}$ Torr. The area of the solar cell was confined to $0.04 \mathrm{~cm}^{2}$.

The current-voltage curve of the cells was measured under illumination with simulated solar light (AM 1.5-100 $\mathrm{mW} \mathrm{cm}^{-2}$ ) from a Kansai Kagakukikai XES-502S. The photocurrent action spectra were measured under illumination with a monochromatic light. In each measurement, the cells were exposed in air, and the illumination was carried out from the ITO side. The ionization potential of the organic solids and electrodes employed in this work was estimated from photoelectron spectroscopy in air (PESA) by a Riken Keiki model AC-2. ${ }^{12}$ 


\section{Results and Discussion}

Figure 2 shows the current-voltage curves of the PV (43 $\mathrm{nm}) /$ P3DT $(62 \mathrm{~nm})$ cell. The thickness of each layer is described in the parentheses, herein and after. The photovoltaic properties were $6.51 \mathrm{~mA} / \mathrm{cm}^{2}$ for the short-circuit photocurrent $J_{\mathrm{sc}}, 0.42 \mathrm{~V}$ for the open-circuit photovoltage $V_{\mathrm{oc}}, 0.36$ for the fill factor $F F$, and $0.99 \%$ for the energy conversion efficiency $\eta$. The $\eta$ value of $1 \%$ was also obtained for the PV/ P3HT cell, summarized in Table 1 . These photovoltaic properties were approximately constant at least for one month with-
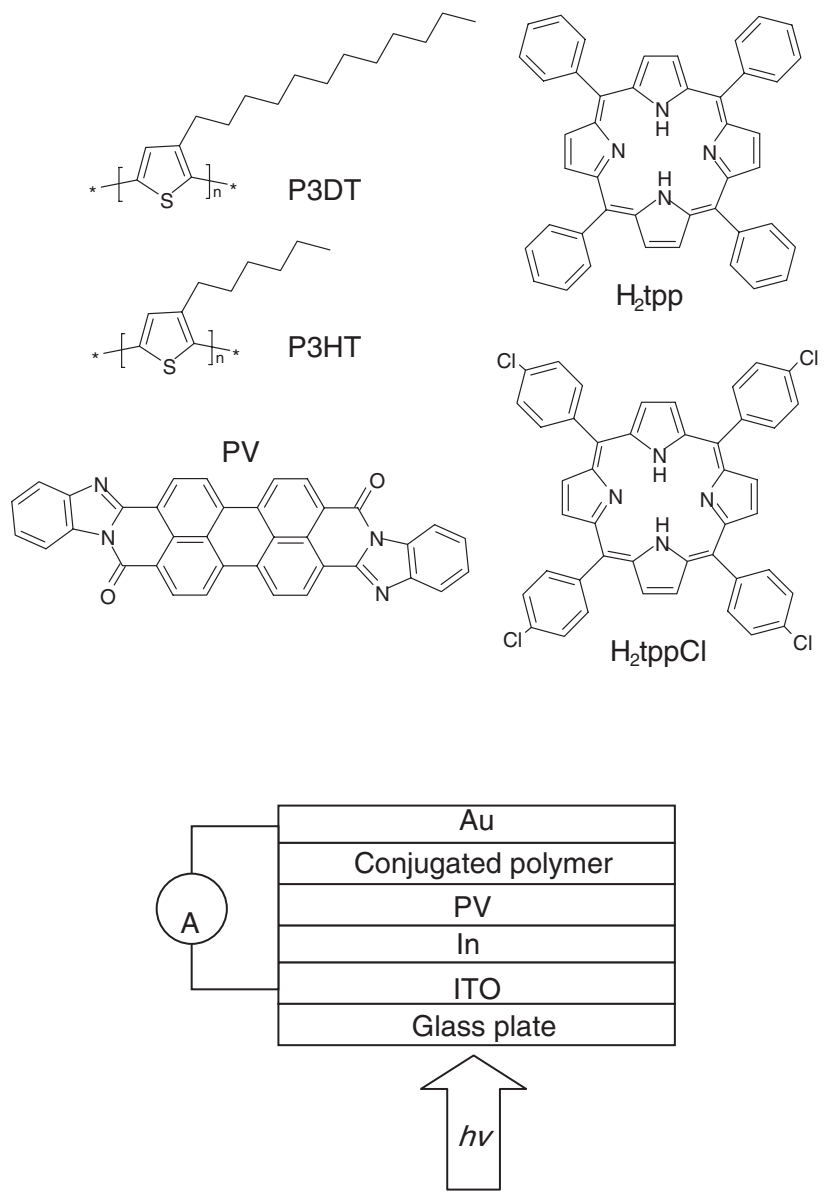

Fig. 1. Structures and abbreviations of organic materials, and structure of $\mathrm{PV} /$ conjugated polymer two-layer photocell. out changing when the devices were stored in air and in the dark. The wavelength-dependence of incident photon to current conversion efficiency, IPCE for the PV/P3DT cell, is shown in Fig. 3, accompanied by the absorption spectra of the PV and P3DT solid films. The photocurrent approximately matched the absorption of PV. However, it reflected only slightly the absorption of P3DT, although this judgment is difficult because of the overlap of the absorption peaks at about $540 \mathrm{~nm}$ of PV and P3DT. Since the fluorescence of the P3DT solid spin-coated onto a vacuum-deposited Au plate was substantially quenched in contrast to that onto a slide

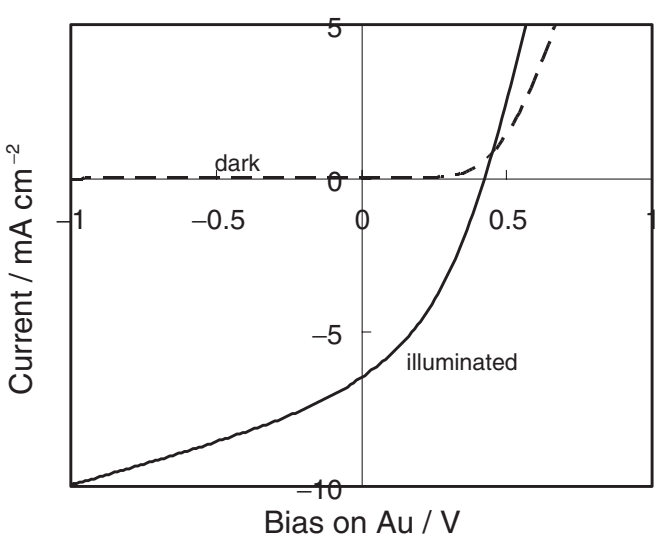

Fig. 2. Photocurrent-voltage curves of ITO/In/PV (34 $\mathrm{nm}) / \mathrm{P} 3 \mathrm{DT}(62 \mathrm{~nm}) / \mathrm{Au}$ solar cell under illumination of AM $1.5-100 \mathrm{~mW} \mathrm{~cm}^{-2}$.

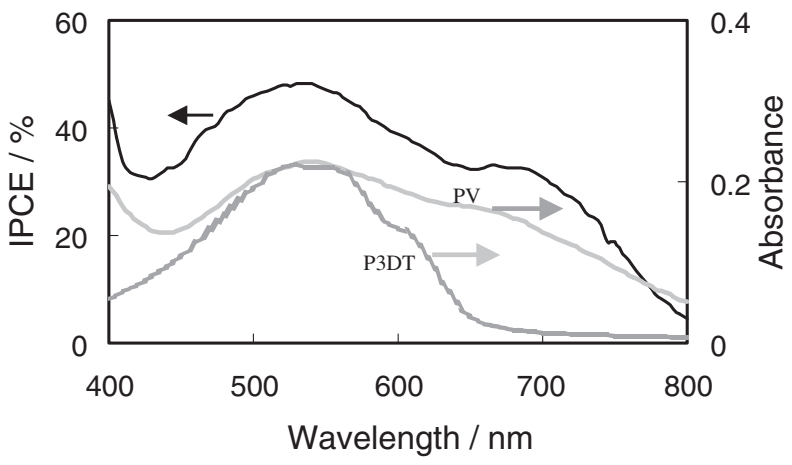

Fig. 3. Photocurrent action spectrum of ITO/In/PV (34 $\mathrm{nm}) / \mathrm{P} 3 \mathrm{DT}(62 \mathrm{~nm}) / \mathrm{Au}$ solar cell, and absorption spectra of PV (34 nm) and P3DT (62 nm) on slide glasses.

Table 1. Performance of PV/Conjugated Polymer Two-Layer Solar Cells under Illumination of AM $1.5-100 \mathrm{~mW} \mathrm{~cm}^{-2}$

\begin{tabular}{ccccc}
\hline Cell structure & $J_{\mathrm{sc}^{\mathrm{a}}}{ }^{\mathrm{a}} / \mathrm{mA} \mathrm{cm}^{-2}$ & $V_{\mathrm{oc}}{ }^{\mathrm{b})} / \mathrm{V}$ & $F F^{\mathrm{c})}$ & $\eta^{\mathrm{d})} / \%$ \\
\hline ITO/In/PV/P3DT/Au ${ }^{\mathrm{e})}$ & 6.51 & 0.42 & 0.36 & 0.99 \\
$\mathrm{ITO} / \mathrm{Au} / \mathrm{PV} / \mathrm{P} 3 \mathrm{DT} / \mathrm{Au}^{\mathrm{f})}$ & 1.48 & 0.38 & 0.38 & 0.21 \\
$\left.\mathrm{ITO} / \mathrm{In} / \mathrm{PV} / \mathrm{P} 3 \mathrm{HT} / \mathrm{Au}^{\mathrm{e}}\right)$ & 4.40 & 0.41 & 0.56 & 1.00 \\
$\mathrm{ITO} / \mathrm{In} / \mathrm{PV} / \mathrm{P} 3 \mathrm{DT}+\mathrm{H}_{2} \mathrm{tpp}^{\mathrm{t}} / \mathrm{Au}^{\mathrm{e}, \mathrm{g})}$ & 3.6 & 0.44 & 0.49 & 0.76 \\
$\mathrm{ITO} / \mathrm{In} / \mathrm{PV} / \mathrm{P} 3 \mathrm{DT}+\mathrm{H}_{2}$ tppCl$/ \mathrm{Au}^{\mathrm{e}, \mathrm{h})}$ & 2.0 & 0.44 & 0.38 & 0.33 \\
\hline
\end{tabular}

a) Short-circuit photocurrent. b) Open-circuit photovoltage. c) Fill factor. d) Energy conversion yield. e) The thickness of In was $5 \mathrm{~nm}$. f) The thickness of Au on the ITO was $10 \mathrm{~nm}$. g) Molar ratio $R$ of porphyrin to thiophene unit in P3DT was 0.08 . h) The $R$ value was 0.12 . 
glass, photoproduced excitons originating from the bulk of P3DT hardly arrived at the charge separation site. Only the excitons near the charge separation site were employed for generating the photocurrent.

The $V_{\mathrm{oc}}$ value of $0.38 \mathrm{~V}$ was observed even in a PV/P3DT two-layer cell sandwiched with an $\mathrm{Au}(25 \mathrm{~nm})$ electrode and an ITO/Au $(10 \mathrm{~nm})$ electrode instead of an ITO/In $(5 \mathrm{~nm})$ electrode, although the $J_{\mathrm{sc}}$ value was small compared to the ITO/In/PV/P3DT/Au cell, shown in Table 1. This means that the photovoltaic effect was observed even when the work function of the current-collecting electrodes was the same. Thus, the photovoltage was not limited by the difference in their work functions. The photovoltage is simply determined here by the built-in potential at the PV/P3DT junction. The limiting factor will be discussed later. Because the contact resistance was large due to non-ohmic resistance between the n-type $\mathrm{PV}$ and the large work functional $\mathrm{Au}$ (as shown later), the $J_{\text {sc }}$ value was much smaller for the ITO/Au/PV/P3DT/Au cell than for the ITO/In/PV/P3DT/Au cell.

The ionization potential $I_{\mathrm{p}}$ of the organic solids and the electrode materials was estimated to investigate the photovoltaic mechanism. The $I_{\mathrm{p}}$ value of P3DT was estimated by the plots of the photoelectron quantum yield from the P3DT solid surface against the incident photon energy. That is, the threshold of the photoelectron quantum yield, as shown by the arrow in Fig. 4, was correlated with the $I_{\mathrm{p}} .{ }^{12}$ The $I_{\mathrm{p}}$ value of other materials was also obtained by this method. The $I_{\mathrm{p}}$ value of the organic solids on ITO was taken as the energy level of the highest occupied molecular orbital (HOMO), and that of the electrode materials as the work function. The energy level of the lowest unoccupied molecular orbital (LUMO) was calculated from both values of the HOMO level and the energy gap estimated from the longest wavelength edge of the absorption spectrum of the solid film. The energy level before contact for the component materials employed in this work is shown in Fig. 5. A hole-transfer from the excited PV to the ground-state P3DT is thermodynamically possible. That is, a large free energy change of $1.2 \mathrm{eV}$ is estimated as the driving force of the photoinduced hole-transfer. Both interfaces of the In/PV and the $\mathrm{Au} / \mathrm{P} 3 \mathrm{DT}$ are taken as ohmic contacts, but the Au/PV interface is considered non-ohmic.

Since it is well known that the PV solid behaves as an ntype semiconductor ${ }^{1}$ and the P3DT solid as a p-type semiconductor, ${ }^{13,14}$ a Fermi-level alignment occurs from the junction of the PV and the P3DT, resulting in the appearance of a space charge, or a built-in potential. The dopant in the P3DT solid may be oxygen molecules physisorbed in the P3DT solid, ${ }^{15-17}$ where the acceptor energy level of the oxygen molecules is perhaps slightly higher than the top of the valence band of P3DT. On the other hand, the dopant in the PV solid is indefinite. Its donor energy level may be slightly lower than the bottom of the conduction band of PV.

We reported previously that the photocurrent in the $\mathrm{Al} /$ P3DT/Au sandwich-type cell was enhanced more than 20 times by blending porphyrin dyes such as $\mathrm{H}_{2}$ tpp and $\mathrm{H}_{2}$ tppCl because of a photoinduced charge transfer between the P3DT and the dye in the Al/P3DT Schottky-barrier. ${ }^{13,14}$ In the blended cell, the discrete pair of hole and electron produced by the photoinduced charge transfer was efficiently separated, result- ing in a large photocurrent. We expected that such a photocurrent enhancement would be observed by blending the porphyrins into the P3DT of the PV/P3DT two-layer cell. However, despite the energy level of PV and porphyrins being suitable for a photoinduced intermolecular electron transfer and an electron transport, as shown in Fig. 5, the photocurrent decreased by blending them as shown in Table 1 . This strongly suggests that the photoinduced charge transfer between the PV and the P3DT was a dominant factor in determining the photocurrent even in the blended two-layer cell. That is, the blended dye merely behaved as an interfering substance for the charge transfer.

From the experimental facts mentioned above, we propose a

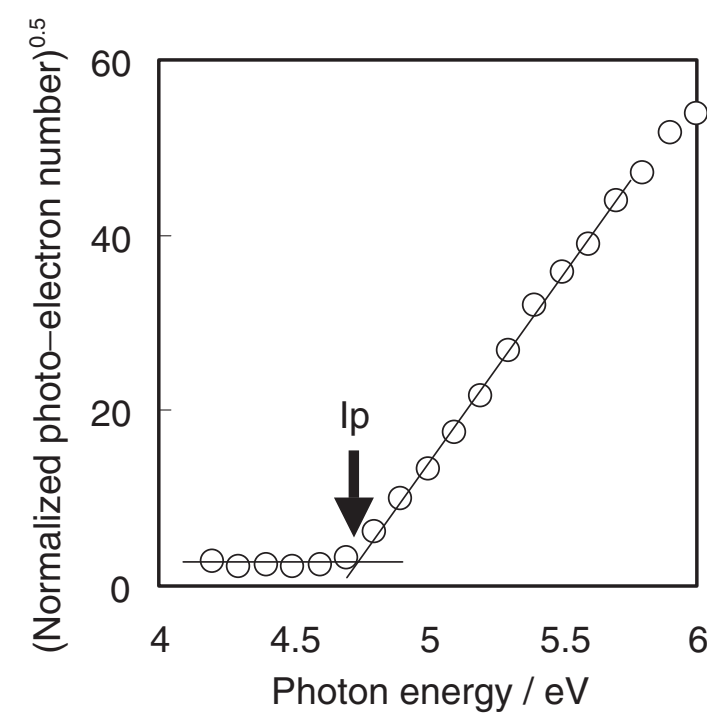

Fig. 4. Normalized photo-electron number ejected from P3DT solid surface against incident photon energy. The P3DT was spin-coated on ITO, and its thickness was 50 $\mathrm{nm}$. The maximum value of incident photon energy was $30 \mathrm{nW} \mathrm{cm}^{-2}$ at $5.9 \mathrm{eV}$. Photon energy for a point of intersection of two linear lines is corresponding to the ionization potential $I_{\mathrm{p}}$.

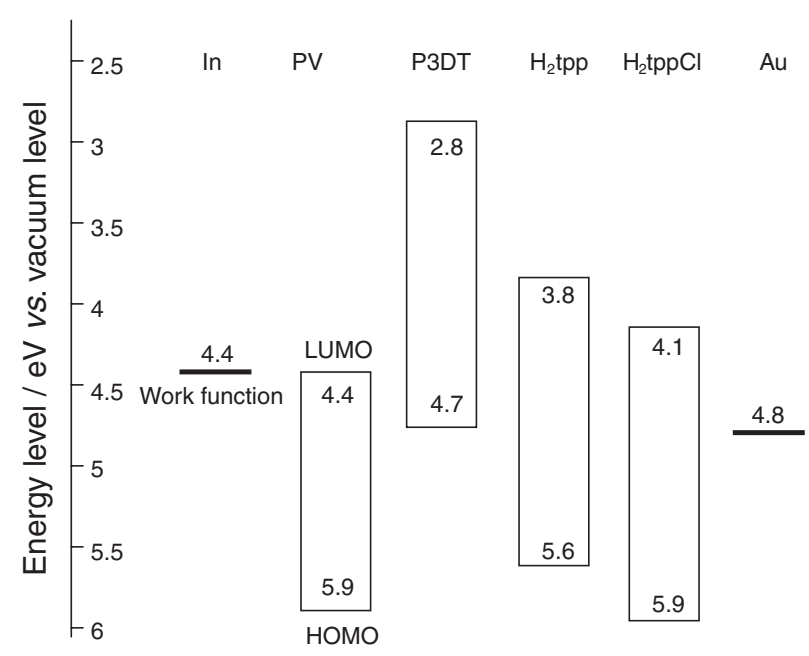

Fig. 5. Energy diagram of the component materials in the organic solar cells. 
photovoltaic mechanism as follows. First, excitons produced by the light absorption of PV migrate to the PV/P3DT interface, typically less than $20 \mathrm{~nm} \cdot{ }^{16,17}$ Secondly, a photoinduced charge transfer between the excited PV and the ground-state P3DT occurs at the PV/P3DT interface, producing the discrete pair of hole and electron. The coulombic force between such an electron and a hole is much smaller than that between the electronhole pair in the PV or P3DT exciton because of the longer distance between them. Hence, the photocharge separation occurs more easily at the PV/P3DT interface with the aid of the builtin potential gradient in the two-layer cell, yielding an enhanced photocurrent. Finally, the free carriers transport to the cathode and the anode, respectively, by the drift caused by the electric field or the diffusion caused by the concentration gradient of the free carriers. The built-in potential is not attributed to the difference in work function of the current-collecting electrodes, as discussed previously, but to the hetero-junction of n-type PV and p-type P3DT. In such a photovoltaic cell, the photoproduced electrons accumulate in the LUMO band of the acceptor PV under an open-circuit condition and the photoproduced holes are accumulated in the HOMO band of the donor P3DT. Consequently, the open-circuit photovoltage is determined by the difference between the LUMO and HOMO levels. However, the $V_{\mathrm{oc}}$ value of $0.42 \mathrm{~V}$ was not consistent with the level difference of $0.31 \mathrm{eV}$. The HOMO and LUMO levels spread somewhat in the solid state. In addition, the orbital density around the top level of the HOMO and the bottom level of the LUMO is small. The ionization via the PESA mechanism occurs from the orbital around the top of the HOMO band, but the position is not always clear. This discrepancy in the $V_{\text {oc }}$ estimation may be ascribed to such a reason.

\section{Conclusion}

The PV/P3DT two-layer cell exhibited a large short-circuit photocurrent of $6.51 \mathrm{~mA} \mathrm{~cm}^{-2}$ under an illumination of AM $1.5-100 \mathrm{~mW} \mathrm{~cm}^{-2}$, resulting in an energy conversion yield of $0.99 \%$. This was because a photoinduced charge-transfer complex consisting of PV and P3DT was efficiently produced at the PV/P3DT interface. Their charge separation then effectively occurred in the built-in potential created by the $\mathrm{p}-\mathrm{n}$ hetero-junction. However, the open-circuit photovoltage of 0.42 $\mathrm{V}$ was small because the difference between the levels of the PV-LUMO and the P3DT-HOMO was small. We believe that further efficient organic solar cells can be prepared by optimiz- ing the electronic interaction between n-type and p-type component materials of a two-layer cell.

This work was supported by the New Energy and Industrial Technology Development Organization (NEDO) under the Ministry of Economy, Trade and Industry (METI).

\section{References}

1 C. W. Tang, Appl. Phys. Lett., 48, 183 (1986).

2 M. Granström, K. Petritsch, A. C. Arias, A. Lux, M. R. Andersson, and R. H. Friend, Nature, 395, 257 (1998).

3 S. E. Shaheen, C. J. Brbec, N. S. Sariciftci, F. Padinger, T. Fromherz, and J. C. Hummelen, Appl. Phys. Lett., 78, 841 (2001).

4 C. J. Brbec, S. E. Shaheen, C. Winder, and N. S. Sariciftci, Appl. Phys. Lett., 80, 1288 (2002).

5 V. D. Mihailetchi, P. W. M. Blom, J. C. Hummelen, and M. T. Rispens, J. Appl. Phys., 94, 6849 (2003).

6 A. J. Breeze, A. Salomon, D. S. Ginley, B. A. Gregg, H. Tillmann, and H.-H. Hörhold, Appl. Phys. Lett., 81, 3085 (2002).

7 M. Hiramoto, H. Fujiwara, and M. Yokoyama, J. Appl. Phys., 72, 3781 (1992).

8 D. Gebeyehu, B. Maennig, J. Drechsel, K. Leo, and M. Pfeiffer, Sol. Energy Mater. Sol. Cells, 79, 81 (2003).

9 P. Peumans, S. Uchida, and S. R. Forrest, Nature, 425, 158 (2003).

10 P. Sullivan, S. Heutz, S. M. Schultes, and T. S. Jones, Appl. Phys. Lett., 84, 1210 (2004).

11 A. D. Alder, R. F. Longo, J. D. Finarelli, J. Assour, and L. Korsakoff, J. Org. Chem., 32, 476 (1967).

12 Y. Nakajima and D. Yamashita, Gekkan Display, 10, 82 (2002).

13 K. Takahashi, T. Iwanaga, T. Yamaguchi, T. Komura, and K. Murata, Synth. Met., 123, 91 (2001).

14 K. Takahashi, M. Asano, K. Imoto, T. Yamaguchi, T. Komura, J. Nakamura, and K. Murata, J. Phys. Chem. B, 107, 1646 (2003).

15 F. J. Kampas, K. Yamashita, and J. Fajar, Nature, 284, 40 (1980).

16 K. Takahashi, J. Nakamura, T. Yamaguchi, T. Komura, S. Ito, and K. Murata, J. Phys. Chem. B, 101, 991 (1997).

17 K. Takahashi, T. Goda, T. Yamaguchi, T. Komura, and K. Murata, J. Phys. Chem. B, 103, 4868 (1999). 